\title{
Scanning X-Ray Nanodiffraction on Living Eukaryotic Cells in Microfluidic Environments
}

\author{
Britta Weinhausen, ${ }^{1}$ Oliva Saldanha, ${ }^{1}$ Robin N. Wilke, ${ }^{1}$ Christian Dammann, ${ }^{1}$ Marius Priebe,${ }^{1}$ \\ Manfred Burghammer, ${ }^{2,3}$ Michael Sprung, ${ }^{4}$ and Sarah Köster, ${ }^{1, *}$ \\ ${ }^{1}$ Institute for X-Ray Physics, University of Göttingen, D-37077 Göttingen, Germany \\ ${ }^{2}$ European Synchrotron Radiation Facility, 6 Rue Jules Horowitz, F-38043 Grenoble, France \\ ${ }^{3}$ Department of Analytical Chemistry, Ghent University, B-9000 Ghent, Belgium \\ ${ }^{4}$ HASYLAB at DESY, Notkestrasse 85, D-22607 Hamburg, Germany
}

(Received 14 November 2013; published 25 February 2014)

\begin{abstract}
High-resolution x-ray imaging techniques offer a variety of possibilities for studying the nanoscale structure of biological cells. A challenging task remains the study of cells by $x$ rays in their natural, aqueous environment. Here, we overcome this limitation by presenting scanning x-ray diffraction measurements with beam sizes in the range of a few hundred $\mathrm{nm}$ on living and fixed-hydrated eukaryotic cells in microfluidic devices which mimic a native environment. The direct comparison between fixed-hydrated and living cells shows distinct differences in the scattering signal, pointing to structural changes on the order of 30 to $50 \mathrm{~nm}$.
\end{abstract}

DOI: 10.1103/PhysRevLett.112.088102

PACS numbers: 87.16.Ka, 87.15.La, 87.16.Ln, 87.59.-e

$\mathrm{X}$ rays are routinely employed to probe the structure and the composition of unlabeled soft matter and biological systems, making use of different techniques like x-ray fluorescence analysis [1], coherent diffractive imaging (CDI) [2-5], or scanning x-ray diffraction with micro- or nanometer-sized beams [6-11]. These methods benefit from the high penetration depth of (hard) x rays, thereby allowing for studies of thick samples while avoiding sample sectioning. Furthermore, the intrinsic small wavelength of $x$ rays provides the possibility to collect structural information with a resolution of tens of $\mathrm{nm}$. In particular, for scanning x-ray diffraction, structural information obtained from individual diffraction patterns in reciprocal space is combined with positional information in real space. Because of the recent availability of small beam sizes in the range of hundreds of $\mathrm{nm}$ and even below [12-14], small sample volumes can be probed to study the local sample structure.

In the past, in most $\mathrm{x}$-ray studies of cellular samples, the specimens were investigated in a dried form, with the advantage of increasing the electron density contrast between the sample and the surrounding medium, leading to a stronger scattering signal. However, the natural environment for most biological systems is aqueous, and consequently, measurements should ideally be performed on fully hydrated samples [15]. Keeping cellular samples in a hydrated environment imposes several challenges on the measurement. Since the electron density contrast is lower, high photon flux synchrotron beam lines are needed to obtain a good signal-to-noise ratio. The sample environment and window material need to be chosen deliberately to be x-ray compatible (resistant to the radiation and avoiding a strong background signal) and suited for cell culture. Silicon nitride $\left(\mathrm{Si}_{3} \mathrm{~N}_{4}\right)$ membranes have proven to be an excellent growth substrate as well as window material [16].

Examples for successful use of $\mathrm{Si}_{3} \mathrm{~N}_{4}$ membranes for cell research have been presented previously. In the soft x-ray regime, imaging of fixed-hydrated and (initially) living eukaryotic cells using scanning transmission $\mathrm{x}$-ray microscopy and spectromicroscopy have been demonstrated [17]. Cellular organelles could be identified and near edge x-ray absorption fine structure spectra were recorded, yielding different calcium concentrations for different cellular regions. Recently, CDI measurements on hydrated yeast cells using $5 \mathrm{keV}$ x rays were presented [15]. Both studies used sealed wet chambers built of two $\mathrm{Si}_{3} \mathrm{~N}_{4}$ membranes. As a further development, we previously presented microfluidic devices employing $\mathrm{Si}_{3} \mathrm{~N}_{4}$ membrane windows as a growth substrate for cells and window material for the $\mathrm{x}$ rays [18]. This approach has the advantage that living cells can be supplied with nutrients during the measurements or manipulating the cells by changing external conditions like $\mathrm{pH}$ or reagent concentration.

Here, we use in situ scanning x-ray diffraction, a labelfree nanoscale imaging technique which - in contrast to most other nanoimaging techniques-does not require fixation of the cells as a prerequisite. Therefore, direct comparison between nanostructures in living and fixed cells and quantification as a function of momentum transfer $q_{r}$ is possible. The small beam (on the order of a few hundred $\mathrm{nm}$ ) enables us to calculate real space x-ray darkfield images of the cells, demonstrating the feasibility of imaging living eukaryotic cells using scanning x-ray techniques. The scattered signal is further analyzed and we obtain distinctly different power law exponents for fixed-hydrated and living cells, respectively. Thus, we 
show an effect of the fixation on the power spectrum which indicates a change in the nanostructure of the cell. We observe structural differences between living and fixedhydrated cells reaching up to length scales of about $60 \mathrm{~nm}$. Thus, the combination of nanoscale resolution in real and reciprocal space provides physical insights which are otherwise not accessible.

Scanning X-ray diffraction experiments are performed at the Göttingen Instrument for Nano-Imaging with X-rays (GINIX) $[19,20]$ at the $P 10$ beam line of the storage ring PETRA III (HASYLAB, DESY, Hamburg, Germany). A schematic of the setup is shown in Fig. 1 and experimental details are described in the Supplemental Material [21]. The radiation is focused by two Kirkpatrick-Baez (KB) mirrors in horizontal and vertical directions, yielding focus sizes of $700 \times 260 \mathrm{~nm}^{2}$ and $390 \times 170 \mathrm{~nm}^{2}$, respectively, (FWHM, horizontal $\times$ vertical) for two different experimental beam times. During the measurements, the fixed-hydrated or living cells are kept in microfluidic devices, which are placed in the focus of the x-ray beam. The primary $\mathrm{x}$-ray beam is blocked by a beamstop and the scattered intensity is recorded on a two-dimensional detector. A schematic of the composition of the microfluidic devices is shown in the inset of Fig. 1. The microfluidic channel is laterally defined by the UV-curable Norland Optical Adhesive 81 and the top and bottom of the channel are closed with $8 \mu \mathrm{m}$ thick Kapton foil [18]. A hole with a diameter of $3.5 \mathrm{~mm}$ is punched through the device in the center of the channel. One side of the hole is closed with an empty $\mathrm{Si}_{3} \mathrm{~N}_{4}$ membrane window and the other side is sealed with a $\mathrm{Si}_{3} \mathrm{~N}_{4}$ membrane window on which the cells are grown. As an example for eukaryotic cells, the cell line SK8/18-2, which was kindly provided by Rudolf Leube (RWTH Aachen, Germany), is employed [22-24].
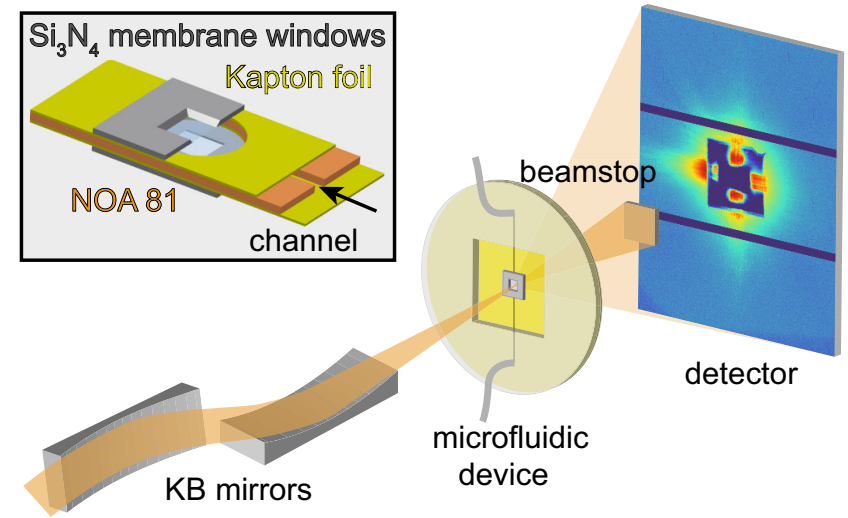

FIG. 1 (color online). Schematic of the measurement setup at the $P 10$ beam line at PETRA III. The $\mathrm{x}$ rays are focused onto the sample using two KB mirrors. Microfluidic devices are used as a sample environment for fixed-hydrated and living eukaryotic cells. The scattered intensity is recorded using a Pilatus $300 \mathrm{~K}$ detector (Dectris Ltd., Baden, Switzerland) (not to scale). The inset shows a detail of the central part of the microfluidic device.
Results of the measurements on living cells are presented in Fig. 2. Figure 2(a) shows a visible light bright-field microscopy image of two living SK8/18-2 taken directly before the $\mathrm{Si}_{3} \mathrm{~N}_{4}$ membrane window is incorporated into the microfluidic device. The microfluidic device is then mounted at the GINIX setup and the cells are inspected with the beam line microscope to confirm that the cells are alive at the start of the measurement. During the measurement, the microfluidic device is flushed with a $\mathrm{CO}_{2}$ independent medium (PAA Laboratories $\mathrm{GmbH}$, Pasching, Austria) supplemented with $10 \%$ fetal calf serum (Invitrogen $\mathrm{GmbH}$, Darmstadt, Germany), $100 \mathrm{U} / \mathrm{mL}$ penicillin and $0.1 \mathrm{mg} / \mathrm{mL}$ streptomycin (Sigma-Aldrich, Munich, Germany). The x-ray dark-field image, which is taken on the marked sample region in Fig. 2(a), is presented in Fig. 2(b). Here, asymmetric step sizes, i.e., $1 \mu \mathrm{m}$ in direction of the fast scan axis (horizontal) and $4 \mu \mathrm{m}$ in direction of the slow scan axis (vertical), with an exposure time of $0.05 \mathrm{~s}$ per position are employed. Compared to taking equal step sizes of $1 \mu \mathrm{m}$ in each direction, this leads to a shorter total scan time for covering the same sample area and a lower average radiation dose per scan point of about $1.8 \times 10^{6}$ Gy $[11,25]$. Furthermore, in exposed regions of the cell, radicals are created. These radicals move
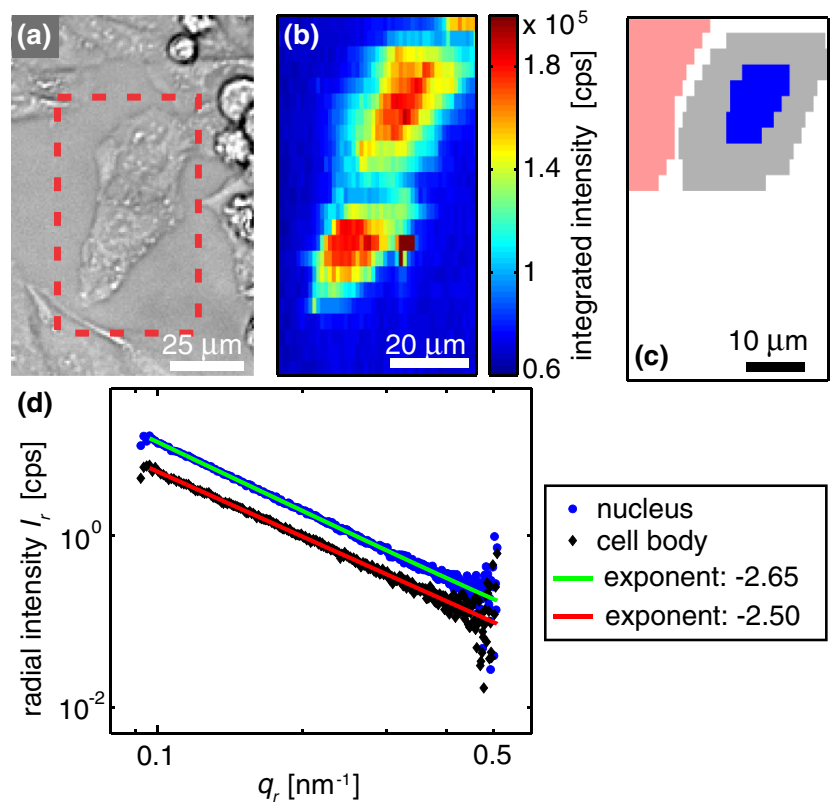

FIG. 2 (color online). Scanning x-ray nanodiffraction measurements on living cells. (a) Bright-field image of two SK8/18-2 cells recorded directly before attachment of the $\mathrm{Si}_{3} \mathrm{~N}_{4}$ membrane window with the cells to the microfluidic device. (b) X-ray dark-field image obtained from a two-dimensional mesh scan with a step size of $1 \times 4 \mu \mathrm{m}^{2}$ on the marked region in panel (a). (c) Selection of three different regions of interest [blue (dark gray): nucleus, light gray: cell body, red (medium gray): empty region]. (d) Fitting of the radial intensity obtained by azimuthal integration of the averaged scattering patterns from different regions of the cell with a single power law function. 
rapidly through the sample and the concentration decreases with distance to the site of creation. Thus, we skip damaged regions by spacing the scan lines (slow scan axis) more widely. In the x-ray dark-field image, the cells can be identified and the nuclei can be distinguished from the cell bodies by a stronger scattering signal, confirming that the cell morphology remains intact during the whole scan.

For further analysis, we compare the scattering signal in the three major regions on the scan: the nucleus, the cell body (of the upper cell), and an empty region as displayed in Fig. 2(c). The scattering patterns recorded in these three regions are averaged and integrated in azimuthal direction. Subtraction of the background signal from the radial intensity profiles obtained on the cell yields the radial intensity profiles in Fig. 2(d). Here, the radial intensities are plotted against the scattering vector $q_{r}$, which relates to real-space sizes $d$ as $d=2 \pi / q_{r}$. The radial intensity from the nucleus is higher than the radial intensity from the cell body, as expected from the x-ray dark-field image. To quantify the intensity decay, power law functions are fitted to the radial intensity profile from the nucleus and the cell body, respectively, and the fit functions are plotted in Fig. 2(d). The power law exponents obtained from the fits are in the range of -2.5 to -3.0 .

For comparison, we also perform measurements on fixed-hydrated cells, which are presented in Fig. 3.
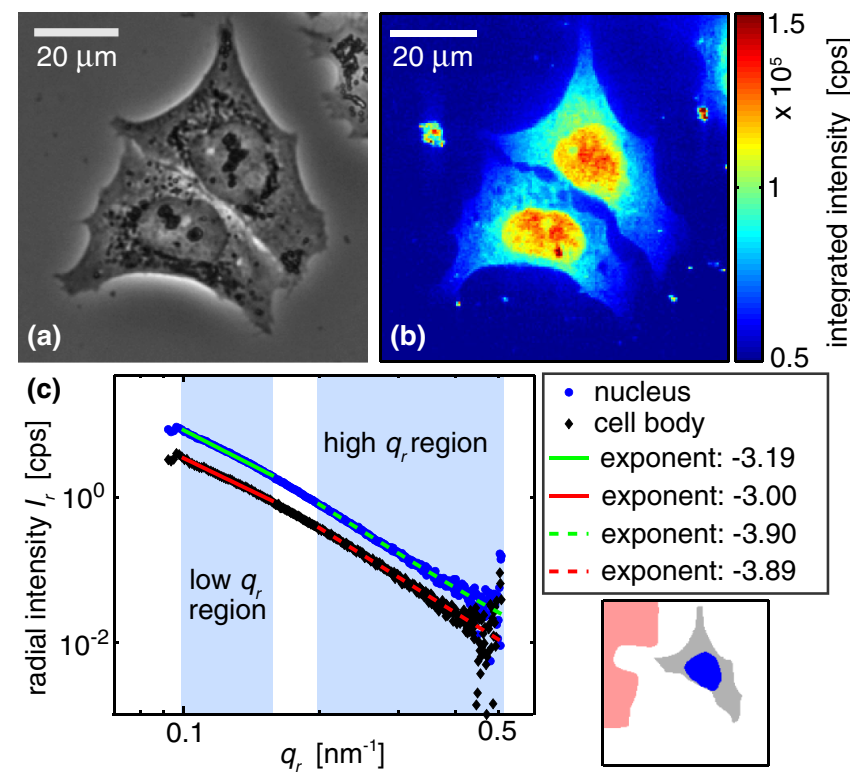

FIG. 3 (color online). Scanning x-ray nanodiffraction measurements on fixed-hydrated cells in a microfluidic device. (a) Visible light phase contrast image of two SK8/18-2 cells after fixation with formaldehyde. (b) X-ray dark-field image obtained from a two-dimensional mesh scan with a step size of $500 \times 500 \mathrm{~nm}^{2}$ on the same sample region as shown in panel (a). (c) Fitting of the high and low $q_{r}$-value regions of the background corrected radial intensity profiles from the nuclear region and the cell body with a power law function. The inset indicates the regions used for averaging.
Figure 3(a) shows a visible light phase contrast microscopy image of two SK8/18-2 cells in buffer after fixation with $3.7 \%$ formaldehyde solution. During the measurements on fixed-hydrated cells, the microfluidic device is flushed with phosphate buffered saline supplemented with $100 \mathrm{U} / \mathrm{mL}$ penicillin and $0.1 \mathrm{mg} / \mathrm{mL}$ streptomycin. On the same sample region, a two-dimensional mesh scan with a step size of $500 \times 500 \mathrm{~nm}^{2}$ and an exposure time of $0.05 \mathrm{~s}$ per position is performed. The average radiation dose per scan point during this scan is estimated to about $2.9 \times 10^{7} \mathrm{~Gy}$. The corresponding $\mathrm{x}$-ray dark-field image is shown in Fig. 3(b). The cells and the cell contours can clearly be identified in the x-ray dark-field image and the cellular morphology agrees with the phase contrast image. Furthermore, the nuclei can be distinguished from the cell bodies. Interestingly, neither the signal from the cell bodies nor from the nuclei is homogeneous but shows substructures. Such features can be found in the visible light micrograph as well, probably corresponding to cell organelles or large vesicles.

In analogy to the measurements on living cells, the scattering patterns from the nucleus and the cell body of the upper cell and an empty region of the scan are averaged and integrated in azimuthal direction. The backgroundcorrected radial intensity profiles from the cell are shown in Fig. 3(c). For the fixed-hydrated cells, the radial intensity profiles exhibit a slight kink at around $0.19 \mathrm{~nm}^{-1}$. Therefore, two power laws are fitted to the low $q_{r}$ region $\left(0.0989-0.1583 \mathrm{~nm}^{-1}\right)$ and high $q_{r}$ region $\left(0.1979-0.5092 \mathrm{~nm}^{-1}\right)$, respectively. The fit functions are plotted in Fig. 3(c) along with the radial intensity profiles. The power law exponents obtained from the fits are in the range of -3.0 to -4.0 with smaller values in the high $q_{r}$ region.

To compare structural features in fixed and living cells, the same analysis scheme is applied to ten fixed-hydrated and eight living cells (see Fig. 4). The power law exponents

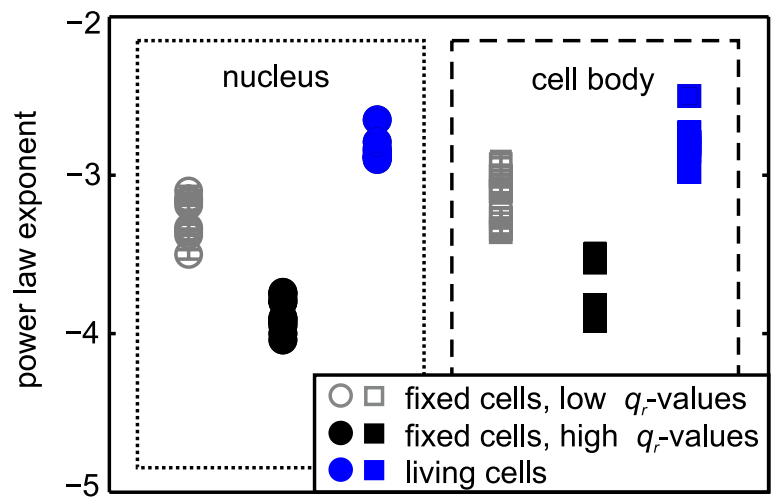

FIG. 4 (color online). Comparison of power law exponents obtained from fits to the background corrected radial intensities for fixed-hydrated and living SK8/18-2 cells. The error bars indicate the errors obtained from the fits with a power law function. 
for the living cells are shown as blue data points, the power law exponents for the fixed-hydrated cells obtained for the low $q_{r}$ region are shown in gray, and the ones for the high $q_{r}$ region in black. The power law exponents obtained for the cell body are slightly higher than the ones obtained for the nucleus. For both regions of the cell, the power law exponents obtained for the living cells are higher than the power law exponents for the fixed-hydrated cells, clearly showing differences between the sample types.

To quantify the changes upon fixation, we compare the radial intensity profiles of fixed-hydrated and living cells. The background corrected radial intensity profiles for the nuclear region and the cell body of each sample type (fixedhydrated or living) are averaged, yielding the profiles shown in Fig. 5(a). The two sets of curves (fixed-hydrated: black, left-hand axis, lower curves, and living: blue, righthand axis, upper curves) are shifted with respect to each other to improve visibility. Here, we use the profiles of six fixed-hydrated and eight living cells recorded with the same experimental parameters, i.e., during the same beam time. Interestingly, from a biophysical point of view, the higher
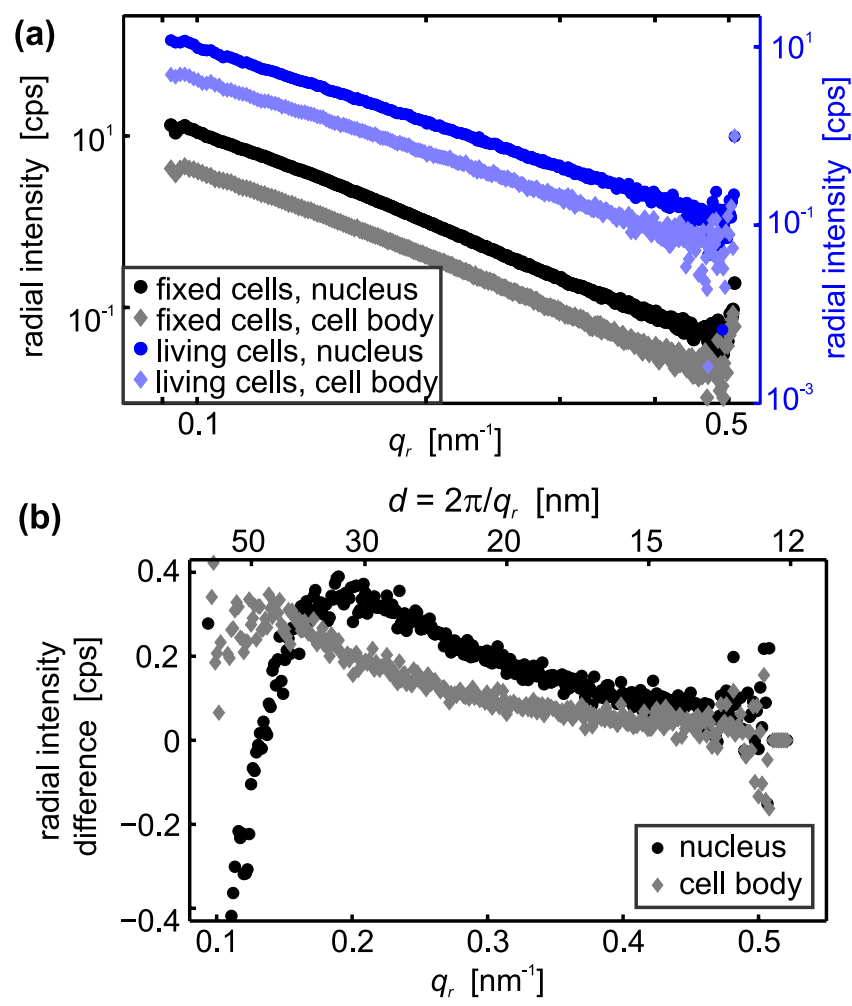

FIG. 5 (color online). Averaged radial intensity profiles for living and fixed hydrated cells. (a) Averaged radial intensity profiles of living and fixed hydrated cells for the nucleus and the cell body after background correction. The two sets of data curves are shifted with respect to each other to improve visibility. (b) Difference of the averaged radial intensity profiles for living and fixed hydrated cells (profile for living cells minus profile for fixed cells). intensities at high $q_{r}$ values indicate a higher imaging resolution for living than fixed-hydrated cells.

The average radial intensity profiles for fixed cells are subtracted from the corresponding profiles for living cells, resulting in the curves presented in Fig. 5(b). Here, the difference curves are plotted against the $q_{r}$ value on a linear scale (bottom axis) and against the corresponding realspace sizes (top axis). Thus, we can specify different regions in the accessible size range. In the difference plot, positive values denote length scales which are more pronounced in the living cells, whereas negative values represent structures that emerge upon fixation. Therefore, aggregates larger than about 40 or $60 \mathrm{~nm}$ for the nucleus and the cell body, respectively, are formed as a consequence of the chemical fixation with formaldehyde, which leads to a cross-linking of the proteins in the cells [26]. Additionally, we observe maxima in the difference curves at about 0.18 and $0.14 \mathrm{~nm}^{-1}$ corresponding to real-space structural sizes of about 35 and $45 \mathrm{~nm}$, respectively. Towards smaller values for $d$, the difference curves decay, and below about $15 \mathrm{~nm}$, no difference is observed.

Comparing cell nucleus and body, both the signals show the same trend. However, the total intensities are shifted to higher values for the nucleus which is likely to be due to the higher packing density of biological matter in the nucleus and the increased amount of material probed by the $\mathrm{x}$ rays at the position of the nucleus. In addition to this shift towards higher intensities, the difference curves for living and fixed cells in Fig. 5(b) are shifted towards higher $q_{r}$ values (smaller characteristic length scales $d$ ) for the (thicker) nucleus as compared to the cell body.

The complex structural arrangements within biological cells cannot be modeled in a straightforward way. A precise and in-depth interpretation of the power law exponents as for solution small-angle $\mathrm{x}$-ray scattering experiments is, therefore, not possible at this time. However, structural differences between living and fixed-hydrated cells reaching up to length scales of about $60 \mathrm{~nm}$ are clearly apparent. As high-resolution visible light microscopy advances, techniques such as stimulated emission depletion (STED) $[27,28]$ can also access these length scales. In STED microscopy, contrary to our approach, specific cellular components are labeled with a fluorescent dye, often after chemical cell fixation, and only these components are visualized. Given the results presented here, the structural alterations upon cell fixation may potentially become apparent in such experiments and might even influence the result or conclusion as compared to living cells.

In summary, we present a novel combination of microfluidics and x-ray nanodiffraction for measurements on whole (living) cells. We demonstrate the utility of the method by imaging eukaryotic cells, providing structural information in real space by $\mathrm{x}$-ray dark-field contrast and in reciprocal space when analyzing the individual diffraction patterns. The alliance of microfluidic devices used for cell 
culture and fast scanning techniques allows for label-free in situ and in operando measurements on living cells, opening up the possibility to perform time-resolved studies and combine cell manipulation and investigation in a straightforward way. The specific application presented here, in addition to providing a solid basis for cellular imaging at high temporal and spatial resolution, shows direct consequences for established experimental methods, such as visible light microscopy. We directly and quantitatively observe that on length scales between about $15 \mathrm{~nm}$ and up to at least $60 \mathrm{~nm}$, cellular nanostructures are altered by chemical fixation using formaldehyde which is a widely used technique in visible light fluorescence microscopy. Consequently, fixation may introduce artifacts. Since recent advances have pushed the resolution limit in visible light fluorescence microscopy well into the size range discussed here, we suggest avoiding chemical fixation if possible or cross-checking potential changes by employing different fixatives when investigating nanometer-sized structures in high resolution visible light fluorescence microscopy.

We thank T. Salditt for fruitful discussions and for help in setting up the instrument; J. Breuer, J.-F. Nolting, F. Westermeier, S. Bondarenko, and A. Zozulya for support during the beam time at the $P 10$ beam line; A. Burkhardt, M. Warmer, and A. Meents for the possibility to utilize the cell culture lab at the $P 11$ beam line; and R. Leube, A. Kölsch, and R. Windoffer for providing the SK8/18-2 cells and for helpful discussions. This work was supported by the German Research Foundation (DFG) in the framework of SFB 755 "Nanoscale Photonic Imaging" and the Excellence Initiative as well as the Helmholtz Gemeinschaft in the framework of Virtual Institute VH-VI403 "In-Situ Nano-Imaging of Biological and Chemical Processes." B. W. is supported by the Deutsche Telekom Stiftung.

*sarah.koester@phys.uni-goettingen.de

[1] T. Paunesku, S. Vogt, J. Maser, B. Lai, and G. Woloschak, J. Cell. Biochem. 99, 1489 (2006).

[2] D. Shapiro, P. Thibault, T. Beetz, V. Elser, M. Howells, C. Jacobsen, J. Kirz, E. Lima, H. Miao, A. M. Neiman, and D. Sayre, Proc. Natl. Acad. Sci. U.S.A. 102, 15343 (2005).

[3] K. Giewekemeyer, P. Thibault, S. Kalbfleisch, A. Beerlink, C. M. Kewish, M. Dierolf, F. Pfeiffer, and T. Salditt, Proc. Natl. Acad. Sci. U.S.A. 107, 529 (2010).

[4] H. Jiang, C. Song, C.-C. Chen, R. Xu, K. S. Raines, B. P. Fahimian, C.-H. Lu, T.-K. Lee, A. Nakashima, J. Urano et al., Proc. Natl. Acad. Sci. U.S.A. 107, 11234 (2010).

[5] R. N. Wilke, M. Priebe, M. Bartels, K. Giewekemeyer, A. Diaz, P. Karvinen, and T. Salditt, Opt. Express 20, 19232 (2012).

[6] P. Fratzl, H. F. Jakob, S. Rinnerthaler, P. Roschger, and K. Klaushofer, J. Appl. Crystallogr. 30, 765 (1997).
[7] H. Lichtenegger, M. Müller, O. Paris, C. Riekel, and P. Fratzl, J. Appl. Crystallogr. 32, 1127 (1999).

[8] O. Paris, Biointerphases 3, FB16 (2008).

[9] B. Müller, H. Deyhle, D. A. Bradley, M. Farquharson, G. Schulz, M. Müller-Gerbl, and O. Bunk, Eur. J. Nanomed. 3, 30 (2010).

[10] O. Bunk, M. Bech, T. H. Jensen, R. Feidenhans'l, T. Binderup, A. Menzel, and F. Pfeiffer, New J. Phys. 11, 123016 (2009).

[11] B. Weinhausen, J.-F. Nolting, C. Olendrowitz, J. LangfahlKlabes, M. Reynolds, T. Salditt, and S. Köster, New J. Phys. 14, 085013 (2012).

[12] H. C. Kang, H. Yan, R. P. Winarski, M. V. Holt, J. Maser, C. Liu, R. Conley, S. Vogt, A. T. Macrander, and G. B. Stephenson, Appl. Phys. Lett. 92, 221114 (2008).

[13] A. Schropp, P. Boye, J. M. Feldkamp, R. Hoppe, J. Patommel, D. Samberg, S. Stephan, K. Giewekemeyer, R. N. Wilke, T. Salditt et al., Appl. Phys. Lett. 96, 091102 (2010).

[14] H. Mimura, S. Handa, T. Kimura, H. Yumoto, D. Yamakawa, H. Yokoyama, S. Matsuyama, K. Inagaki, K. Yamamura, Y. Sano et al., Nat. Phys. 6, 122 (2010).

[15] D. Nam, J. Park, M. Gallagher-Jones, S. Kim, S. Kim, Y. Kohmura, H. Naitow, N. Kunishima, T. Yoshida, T. Ishikawa et al., Phys. Rev. Lett. 110, 098103 (2013).

[16] E. A. Carter, B. S. Rayner, A. I. McLeod, L. E. Wu, C. P. Marshall, A. Levina, J. B. Aitken, P. K. Witting, B. Lai, Z. Cai et al., Mol. Biosyst. 6, 1316 (2010).

[17] J. Sedlmair, S.-C. Gleber, S. O. Mert, M. Bertilson, O. von Hofsten, J. Thieme, and T. Pfohl, Microsc. Microanal. 17, 991 (2011).

[18] B. Weinhausen and S. Köster, Lab Chip 13, 212 (2013).

[19] S. Kalbfleisch, H. Neubauer, S. P. Krüger, M. Bartels, M. Osterhoff, D. D. Mai, K. Giewekemeyer, B. Hartmann, M. Sprung, and T. Salditt, AIP Conf. Proc. 1365, 96 (2011).

[20] T. Salditt, S. Kalbfleisch, M. Osterhoff, S. P. Krüger, M. Bartels, K. Giewekemeyer, H. Neubauer, and M. Sprung, Opt. Express 19, 9656 (2011).

[21] See Supplemental Material at http://link.aps.org/ supplemental/10.1103/PhysRevLett.112.088102 for further information concerning the cellular sample preparation and the fabrication of microfluidic devices as well as the x-ray measurements and the data treatment.

[22] P. Strnad, R. Windoffer, and R. E. Leube, J. Cell Sci. 115, 4133 (2002).

[23] R. Windoffer, S. Wöll, P. Strnad, and R. E. Leube, Mol. Biol. Cell 15, 2436 (2004).

[24] S. Wöll, R. Windoffer, and R. E. Leube, Eur. J. Cell Biol. 84, 311 (2005).

[25] M. R. Howells, T. Beetz, H. N. Chapman, C. Cui, J. M. Holton, C. J. Jacobsen, J. Kirz, E. Lima, S. Marchesini, H. Miao et al., J. Electron Spectrosc. Relat. Phenom. 170, 4 (2009).

[26] J. A. Kiernan, Microsc. Today 8, 8 (2000).

[27] S. W. Hell and J. Wichmann, Opt. Lett. 19, 780 (1994).

[28] L. Meyer, D. Wildanger, R. Medda, A. Punge, S. O. Rizzoli, G. Donnert, and S. W. Hell, Small 4, 1095 (2008). 